\title{
Study on Intelligent Speed Control Algorithm for Diesel Engine
}

\author{
Cheng Ma, Enzhe Song, Guofeng Zhao and Chong Yao \\ *Department of power and energy engineering, Harbin Engineering University, Harbin, 150001, China \\ *Corresponding author. Email:sez2005@sina.com
}

\begin{abstract}
Synopsis
In this paper, two types of intelligent controllers are designed based on the RBF neural network algorithm and active disturbance rejection control (ADRC) technology to solve the problem that the dynamic speed is difficult to control for diesel engine. In order to verify the speed regulation performance of the intelligent control system a mean value modeling (MVM) of D6114 generation diesel engine was established for off-line simulation, and the above two intelligent algorithms were compared with PID. The results show that the ADRC has a relatively small overshoot and quick dynamic response for diesel engine speed control. Radial basis function (RBF) intelligent algorithm can real-timely optimize the control parameters and has good adaptability in speed control, the transient rate decreased by $1.6 \%$ and stable time is shortened by $1.46 \mathrm{~s}$ compared with common PID algorithm. The control performance under condition of start-up, idle speed and mutation load is compared. The results show that RBF neural network controller has good learning and adaptive capabilities for speed control of diesel engine. It can balance the stability at different speed and output of large rack displacement in a short time when the load changes to reduce the influence of load change on the rotational speed. For ADRC controller, it maintains good effect when the nonlinearity in the system increases. Improvement of PID using TD has fast response at startup and under disturbances. With NLSEF and ESO, NLSEF can automatically adjust the output according to the speed deviation to reduce interference while ESO can correct the control amount to improve the control effect of load change.
\end{abstract}

Keywords: RBF; ADRC; Speed control; Diesel engine

\section{Introduction: Research adaptive control algorithm}

Diesel engines are being paid more attention due to their natural high efficiency and low fuel consumption which are widely used for a wide spectrum of industrial applications and for electric power generation systems. Despite several of their attractive features, diesel engines are subject to highly nonlinear and time varying dynamics which make them hard to control. In order to get the best performance, speed controller is essential in speed regulation, robustness to load disturbance and fuel efficiency.

However, experiment shows that traditional fixed-gain PID must provide a compromise of engine performance during both steady-state and transient load disturbances (David J. Mcgowan and D. John Morrow,

Dr. Song is professor of Energy and Power Engineering, Harbin Engineering University. His research interest includes Electronic Control of Fuel Injection and Speed Control Technology, Dual Fuel Engine Electronic Control Technology, Intelligent Control System Design and Development.

Mr. Ma is a doctoral candidate in Harbin Engineering University, China and his major is Marine Engineering. His research focus is Engine Intelligent Control and Advanced Control Algorithm.

Dr. Yao is an associate professor of the Energy and Power Engineering, Harbin Engineering University. His research interest includes Control System Integration Development, System Performance Comprehensive Optimization.

Mr. Zhao is a doctoral candidate in Harbin Engineering University, China and his major is Marine engineering. His research focus is Engine Control System Development and Performance Optimization. 
2003). Furthermore, traditional PID controller needs tedious experiments to calibrate the control parameters. As is well known, in operating condition the performance of diesel electronic governor is often affected by the environmental factors such as temperature, atmospheric pressure and the diesel itself. Marine Diesel Engine for ship's propulsion is a highly complex process that is not easy to describe mathematically, which makes the design of reliable controller very difficult (Petar Matic and Nikola Račić, 2009).

So there is a badly need of a self-adaptive controller. In recent years, the emergence and rapid development of artificial intelligence has provided a new method for the control field, and the intelligent PID has become a new research hotspot and development direction. Neural networks can automatically learn plant models and are used for control immediately, so the application of neural networks to recognition and adaptive control has always aroused great interest. S. Roy et al. proposed an adaptive control scheme that uses adaptive controllers to solve the problem of nonlinear and time-varying characteristics of diesel generator dynamic speed control (S. Roy, and O. P. Malik et al, 1991). And they apply the mentioned algorithm to Texas instruments' DSP 320 C30 to test the control effect (S. Roy, and O. P. Malik, et al, 1993). F. Karray et al. also mentioned the speed control method (F. Karray, and E. Conrad, 1997). In this paper, they have discussed gain scheduling, Fuzzy logic technique, neural network and neuro-fuzzy controller. Moreover J. S. Ha et al. design an intelligent speed control system for marine diesel engine. The approach is adopted to use a conventional PID controller for normal operation and a neural network feedforward controller for adaptive control. The simulation results show the applicability of the scheme in diesel speed adaptive control (J. S. Ha, and S. J. Oh, 1997). Professor Guo et al. also studied the intelligent algorithm apply in ship diesel engine speed control system (J. H. Guo and S. H. Liang, et al, 2006), a new intelligent PID control algorithm based on CMAC neural network, using its self-learning ability online setting controller parameters, the simulation shows that the algorithm has high precision and strong adaptability. Furthermore, a double-pulse $H_{-} \infty$ is used to propose a feedback function to restrain the load disturbance and improve the dynamic precision of the diesel engine (H. G. Lei and W. C. Hong, 2007). In addition to an on-line learning and self-tuning error model to improve the speed of the regulator is discussed (Guichen. Z and Guang. R, 2009), the control scheme is based on back propagation multi-layer perceptron adaptive neural network in which a modified hyperbolic tangent function is used as the activation function of an auto-tuning neuron, and updated by the fastest descent method. Then a neural adaptive controller based on system recognition and mode control theory is proposed by Jian Z. David J. McGowan introduced integrated fuzzy logic control in $33 \mathrm{~kW}$ standby diesel generator set, and unload voltage fluctuation is $5.5 \%$, adjust time is 1.558s (David J. McGowan, and Morrow, D. J. 2006). Additionally Naeim Farouk Mohammed (2013) simulated genetic algorithm and PID on D6114. Fuzzy-PID with multiple input control on a same diesel the adjust time is 1.34s (David J. McGowan and Morrow, D. J. 2008). Yufeng Zhang (2012) has used fuzzy self-tuning PID to improve the quality of power supplied by medium and small size diesel generators. Chen Ailing (2010) developed a model free adaptive (MFA) controller for diesel speed regulation. Moreover Shi Yong (2013) introduced two kinds of neural networks to control the position loops and speed loops, and BP neural network is used to control the rack position actuator (Yong S. and Da Z. Q. 2013). And Sun, JB also has built a neural network simulation system for large low speed two-stroke diesel; the results show that the neural network can improve the dynamic response of propulsion system. E. Mesbahi (2003) simulates the offline neural network identification and control technique confirming that ANN can capture dynamic behavior of plant and can respond correctly to unseen/untrained signal. However, when the system has great uncertainty, BRF neural network can effectively improve the performance of the controller. In addition, it can approximate any nonlinear function with any precision, and has the ability of global approximation. It fundamentally solves the local optimal problem of BP network. Moreover, the topology structure is compact, and the structural parameters can be separated and learned quickly (Girosi, F., and T. Poggio, 1990). 
For the nonlinear characteristics of the Marine engine speed regulation system and influenced by factors such as wind, wave and flow, the engine speed is difficult to control, so many scholars studied its control method. Pan weigang et al. present an active disturbance rejection nonlinear control strategy, and the fuzzy controller is used to modify parameters of ADRC online to improve the ADRC's adaptive capacity. Besides a ship main engine fuzzy adaptive ADRC controller is designed (Wei G. P. 2010). At the paper of (Wei G. P. et al. 2010) they used the expert system to modify parameters of ADRC, and an optimization objective function was proposed to modify ADRC parameters online by genetic algorithm. And also a ship course genetic algorithm ADRC controller is designed (Weigang P. and Xiao H. 2015, Yingbing Z.). Moreover an idle speed controller was designed by an active disturbance rejection control framework, which to compensate for varying engine loads and friction torque in passenger car diesel engines (Kang, E., S. Hong, and M. Sunwoo, 2016).

The main contribution of this paper is to explore some solutions to the effective speed regulation based on RBF-PID and ADRC, which are self-adaptive control schemes. RBF-PID is truly applied in diesel governor and developed the controller. Bench experiments have carried out using the designed controller. Combining RBF neural network and PID the new control strategy is designed. A MTALAB/SIMULINK model is built to validate its feasibility. Neural network self-adaptive controller software is developed, and its feasibility was validated by experiments. And also developed an ADRC based on the Simulink module, that consist of tracking differentiator (TD), nonlinear states error feed-back (NLSEF) and extended state observer (ESO) module. An improved PID model is established by using the TD to improve the performance of traditional PID control algorithm.

\section{Mathematical modelling and simulation}

The control-oriented models (COM) should be simple enough to run fast while accurate enough to be reliable. Computer simulation is an effective way to shorten the development cycle of new control strategy. Off-line simulation needs a suitable model of the control system which consists of controller, controlled object and the actuator. Mean value model is established and S-function are used to achieve core intelligent control algorithm. Mean value model (MVM) of diesel engine has been widely disgusted, so the detail about diesel model will not be stated. But to increase the accuracy of the model many works have been done. The principle of engine speed control system is shown in firgur1, and the mathematical model is established according to the working principle and the system structure of the engine.

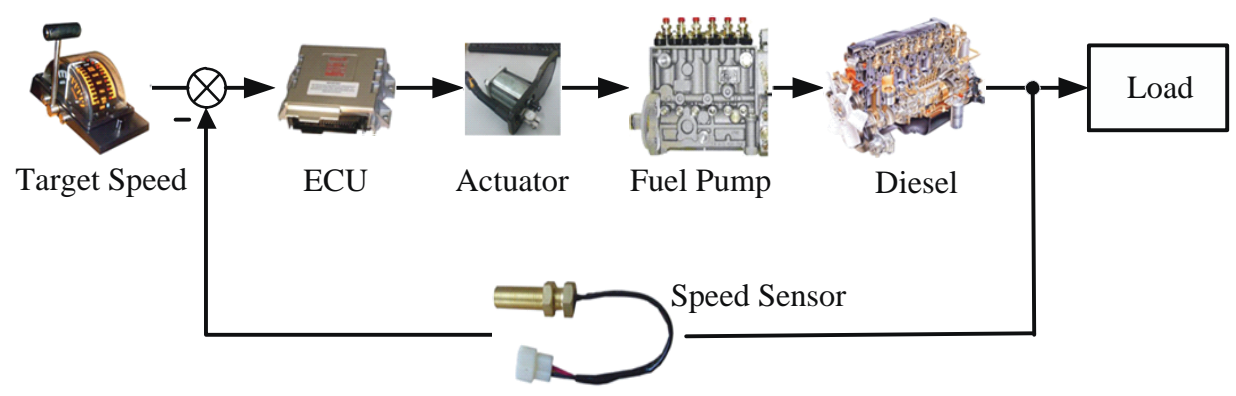

Figure 1 Schematic diagram of engine speed control system

\subsection{Mean value model of diesel engine}

The MVM of D6114 (a high speed diesel engine) is established which consists of following sub-models: volumetric efficiency model, air intake flow model, indicated thermal efficiency model and diesel dynamic model. Volumetric efficiency is a function of speed. Indicated thermal efficiency is a function of air-fuel ratio.

Where qmf module is used to calculate the amount of oil injected into the cylinder per unit time. According 
to the relationship between $q m f$, low caloric value of diesel $\left(H_{u}\right)$, indicated thermal efficiency $\left(\eta_{i}\right)$ and engine speed $(n e)$, the indicated torque of engine is calculated. The friction torque is summarized by empirical formula. The yitav module represents cylinder charging efficiency that can be described as a function of engine speed. Air mass flow rate is calculated according to the engine speed and efficiency of the cylinder. The simulation model of diesel engine is shown in Figure2.

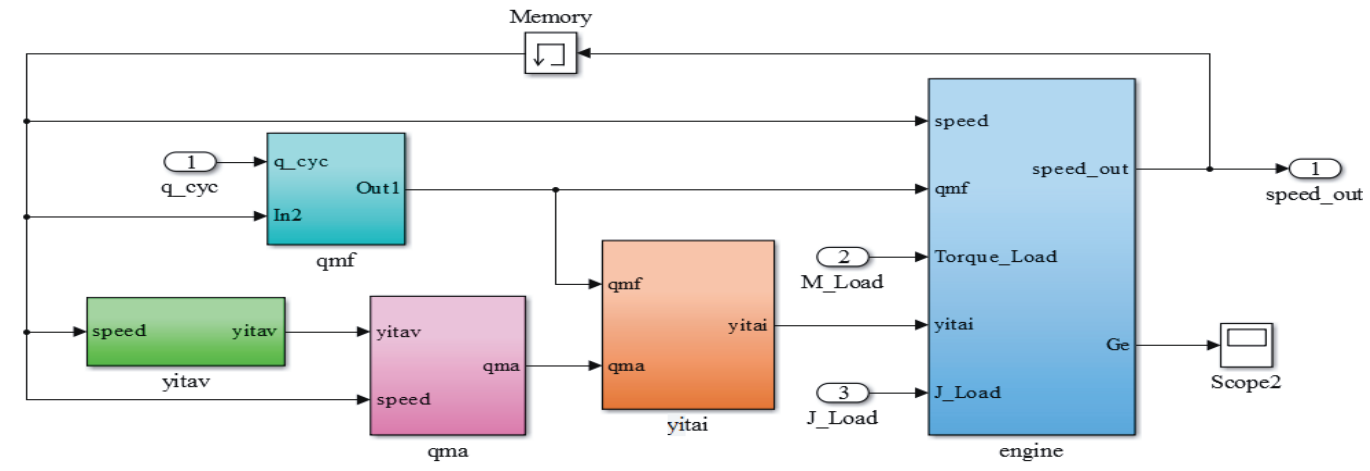

Figure 2 Model of diesel engine

\subsection{Actuator model}

The displacement actuator is driven by a proportional electronic magnet, which has strong anti-interference, good dynamic and quick response characteristics. Its mathematical model is simplified as a transfer function:

$$
G(s)=\frac{k}{T_{k} s+1}
$$

The simulation model of electromagnetic actuator is shown in Figure3.

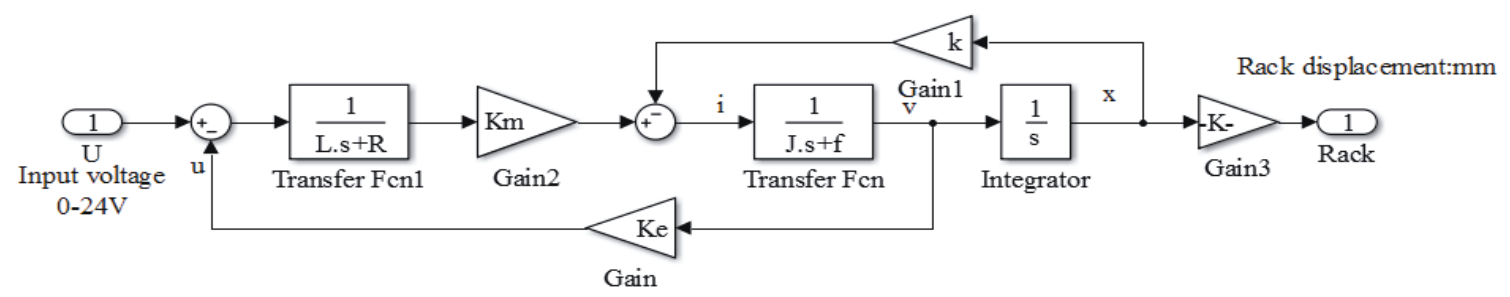

Figure3 Model of electromagnetic actuator

\subsection{Fuel injection pump model}

The accuracy of fuel injection pump model directly affects the overall performance of the diesel engine speed regulation system. In this paper, the model of injection pump is established based on experimental data. First record the rack displacement when diesel speed keeps at 1500r/min and the load ranges from 0 to $100 \%$.

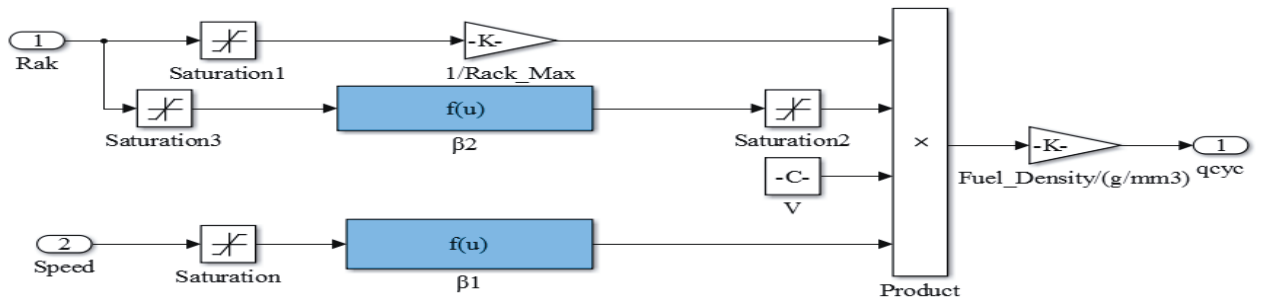

Figure4 Fuel injection pump model

Second the load is kept as a constant and the speed ranges from 700r/min to $1500 \mathrm{r} / \mathrm{min}$, then record the rack displacement. By fitting the test data, the velocity characteristic coefficient of the high-pressure oil pump $\beta_{1}$ 
and the position characteristic coefficient of the rack $\beta_{2}$ were obtained.

\section{Design and structure of intelligent control system}

\subsection{The diesel engine RBF-PID controller design}

Closed loop controller structures are used extensively in the structure of engine speed control system. In this paper, the rotation speed of crank shaft and rack displacement of injector pump is used for double closed loop. The traditional PID control algorithm is used to control the rack position because of the linearity of the electromagnetic actuator is perfect and it is not easily affected by environmental factors. Dynamic speed control of diesel engine is difficult due to the extremely nonlinear, time-varying constants. RBF neural network has strong generalization ability and simple structure; it can approximate any nonlinear curve with any accuracy, so it's learning speed is fast, which can meet the real-time requirements. So a RBF-PID controller is used for speed regulation. In this paper, the neural network structure consists of an input layer composed of speed, speed deviation and rate of change of deviation; an output layer with $K_{P}, K_{i}$, and $K_{d}$ and the hidden layer form a three-layer structure. The gradient descent method was selected to update the weight value, and the attenuation speed of the curve was adjusted by adjusting the width of Gaussian basis function.

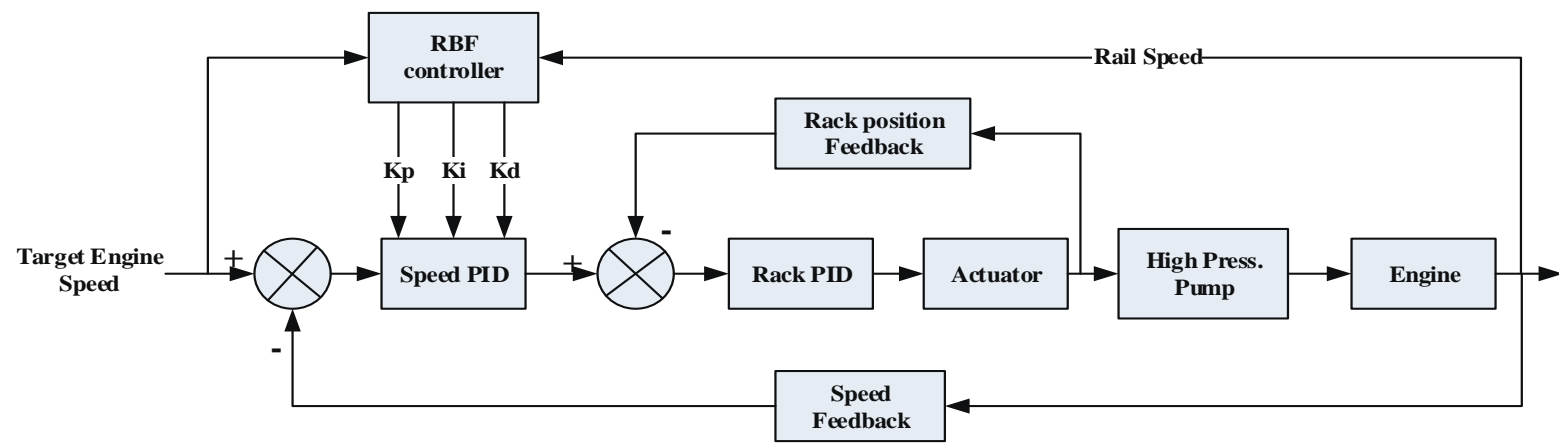

Figure 5 The structure of RBF-PID controller

\subsection{Design of ADRC controller}

\subsubsection{Structure of the ADRC controller}

The three main parts of ADRC (TD-nonlinear tracking differentiator, ESO- extended state observer and NLSEF-nonlinear state error feedback control law) can be seen in Figure6. The ultimate goal for designed the diesel engine controller is to maintain the accuracy and tracking to speed, so the differential signal can be tracked reasonably by TD module and NLSEF module is used to process the error signal to improve the performance of the controller. Where $n_{s}$ is expectations of the engine speed, $x$ is the actual displacement of the actuator, $n_{e}$ is the actual speed of the engine. 


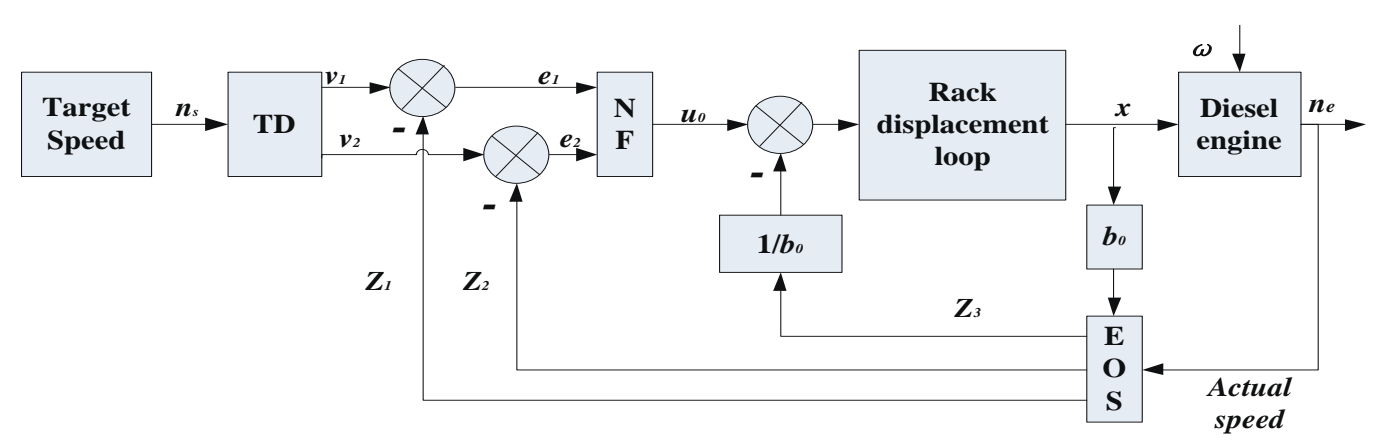

Figure 6 ADRC controller for speed regulation

Where $v_{2}$ is differential signal of $n_{s}, z_{1}, z_{2}$ are the estimation of output $n_{e}$ and its differential, $z_{3}$ is the estimation of total disturbance; $e_{1}, e_{2}$ are the error, $u_{0}$ is the output of NF.

\subsubsection{Parameter features analysis of ADRC}

ADRC has a definite structure, so its performance mainly depends on the parameter setting. There are 8 parameters of the ADRC controller should be set by analysis.

The parameters $\left\{\beta_{01}, \beta_{02}, \beta_{03}\right\}$ are determined by the sample period of the controller. In this case, the parameters of the controller that needs to be adjusted are the gain $r$, the damping coefficient $c$, the precision factor $h_{1}$ and the compensating factor $b_{0}$. The bigger $r$ is, the faster the tracking is and the higher system's execution ability of the control objects. The remaining three parameters are similar to the $K_{P} 、 K_{i}$, and $K_{d}$ in the PID controller.

\section{Experiment result analysis and discussions}

\subsection{RBF-PID controller}

\subsubsection{Dynamic performance comparison}

As an important indicator of diesel engine control performance, dynamic characteristics are analyzed by means of sudden $100 \%$ load and reduction. Furthermore, the dynamic performance of the engine is compared with that of traditional PID, BP and RBF neural network. Sudden add 100\% load at 40s and remove all load at $20 \mathrm{~s}$ later, the following simulation results are obtained. The sampling time is set to $0.002 \mathrm{~s}$ in the simulation model.

Table1: Compare for the Simulation Results of Speed Governing Performance

\begin{tabular}{ccccccc}
\hline & \multicolumn{2}{c}{ Traditional PID } & \multicolumn{2}{c}{ BP-PID } & \multicolumn{2}{c}{ RBF PID } \\
\cline { 2 - 7 } & $\begin{array}{c}\text { Sudden add } \\
\text { 100\% load }\end{array}$ & $\begin{array}{c}\text { 100\% load } \\
\text { reduction }\end{array}$ & $\begin{array}{c}\text { Sudden add } \\
\mathbf{1 0 0 \% ~ l o a d ~}\end{array}$ & $\begin{array}{c}\text { 100\% load } \\
\text { reduction }\end{array}$ & $\begin{array}{c}\text { Sudden add } \\
\text { 100\% load }\end{array}$ & $\begin{array}{c}\text { 100\% load } \\
\text { reduction }\end{array}$ \\
\hline Peak speeds(r/min) & 1407 & 1593 & 1424 & 1577 & 1428 & 1572 \\
Transient rate (\%) & 6.2 & 6.2 & 5 & 5.1 & 4.8 & 4.8 \\
Stable time(s) & 2.45 & 2.54 & 1.74 & 1.7 & 1.39 & 1.52 \\
\hline
\end{tabular}

The speed governing performance simulation results of the three control algorithms are shown in Table1. Under the condition that the three algorithms meet the secondary precision of Marine power station, the BP and RBF intelligent algorithm are better than the traditional PID algorithm in speed control effect. And the two kinds of intelligent control algorithm are better than traditional PID in the stable time and transient rate in the condition of sudden load change. Moreover, the RBF intelligent algorithm has a slight advantage over the BP neural network in the speed stabilization time, but the advantages of the transient rate are not obvious. So more research is being done to solve this problem. 
Under the optimal initial weight condition on the test stand, the start-up, the sudden add $100 \%$ load and load decrease test were performed to verify the effectiveness of RBF-PID speed control algorithm. The comparison between simulation and test results is shown in Figure7 (a) (b) (c), and the comparison between RBF-PID and traditional-PID algorithm is shown in Figure7 (d) (e) (f). The test data is shown in Table 2.

In the start-up process, it can be seen from Figure7 (d) that the overshoot of engine speed is smaller and the stability is better. In the case of load mutation, the neural network can optimize the control parameters in real time according to the change of rotational speed, and the transient rate is reduced about $1.3 \%$ compared to that of traditional PID, and the stable time decreases about 1 second.

Table2 Compare for the Experiment Results of Speed Governing Performance

\begin{tabular}{ccccc}
\hline & \multicolumn{2}{c}{ Traditional PID } & \multicolumn{2}{c}{ RBF-PID } \\
\cline { 2 - 5 } & $\begin{array}{c}\text { Sudden add } \\
\text { 100\%load }\end{array}$ & $\begin{array}{c}\text { 100\% load } \\
\text { reduction }\end{array}$ & $\begin{array}{c}\text { Sudden add } \\
\text { 100\%load }\end{array}$ & $\begin{array}{c}\text { 100\% load } \\
\text { reduction }\end{array}$ \\
\hline Peak speed(r/min) & 1406 & 1598 & 1426 & 1579 \\
Transient rate (\%) & 6.27 & 6.53 & 4.93 & 5.27 \\
Stable time(s) & 3 & 3.2 & 2.1 & 2.2 \\
\hline
\end{tabular}
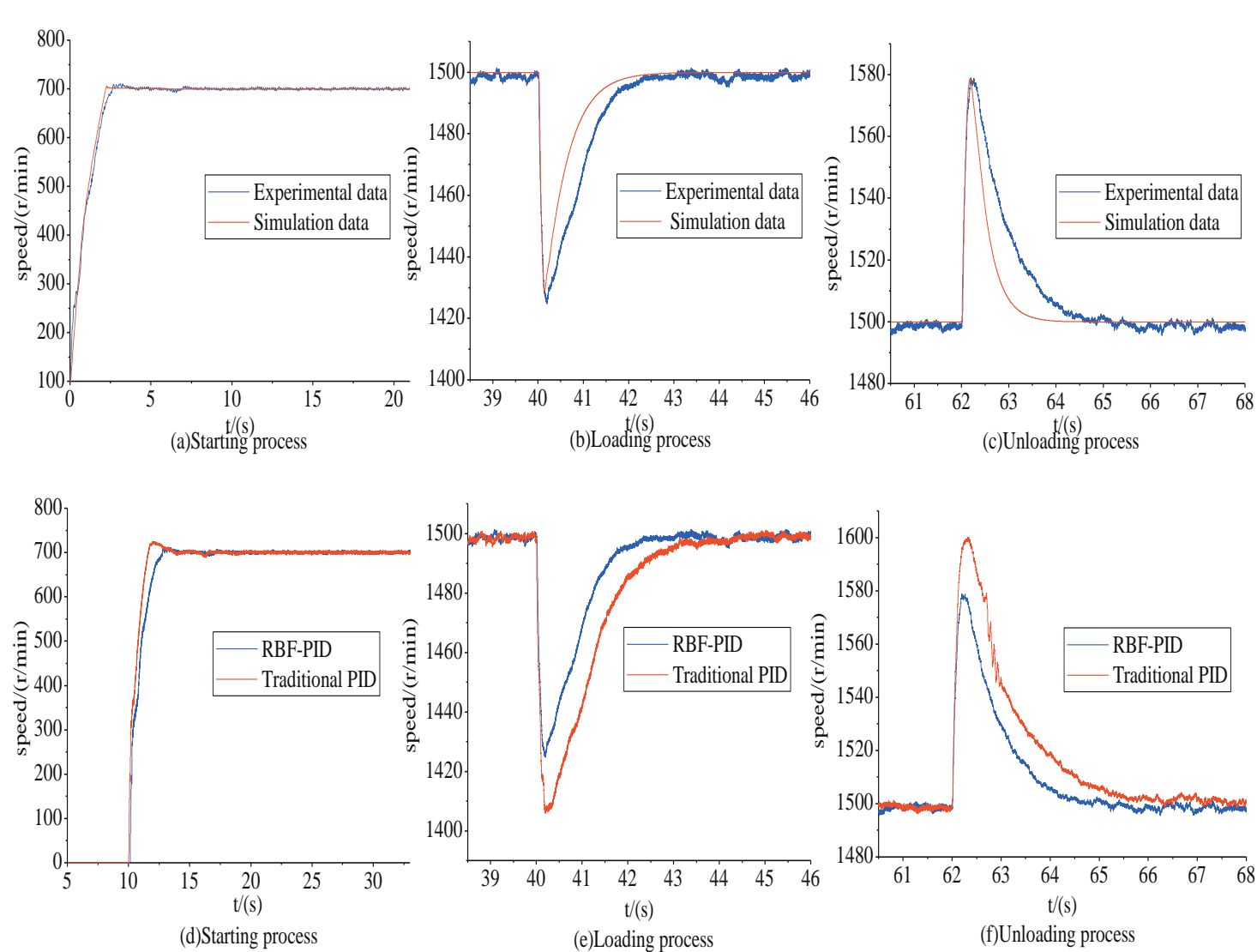

Figure 7 Experimental data comparison for RBF-PID and Traditional-PID algorithm

\subsubsection{Robustness contrast}

A further comparison on the robustness is necessary because of the advantages of the transient rate are not obvious between BP and RBF. In order to compare the influence of initial weights of two intelligent control algorithms, multiple groups of different initial weights were randomly selected for simulation under load mutation conditions. The speed curves of two intelligent algorithms are observed in 5 groups, which are the most 
representative (with the best effect, the longest stable time and the maximum transient rate et al). The comparison of speed regulation performance of two intelligent algorithms is shown in Figure8. The case of (a) (c) in Figure8 is showing the revolution curve of 5 sets of different weights under the BP-PID control algorithm, and the case of (b) (d) is under the RBF-PID control algorithm.

As can be seen from Figure8, when the initial weight changes, the speed stabilization time and overshoot of the two different control algorithms will changes in the process of load change; the optimal initial weight value obtained from the experimental calibration starts to change the value of it gradually, and the speed control effect of the two control algorithms is varying to varying degrees. However, the control effect of BP-PID algorithm has a great change, by contrast, although the control effect of RBF-PID algorithm has also changed, but it's small, and can also keep the diesel engine running stabile within the range of acceptable.

So we can conclude that the BP-PID control algorithm is more dependent on the initial weight of the calibration than RBF-PID. When the external conditions change, by using the BP neural network algorithm it is necessary to adjust the initial weight to meet the control requirements, but RBF-PID algorithm is not used. It is shown that RBF-PID control algorithm has better robustness.

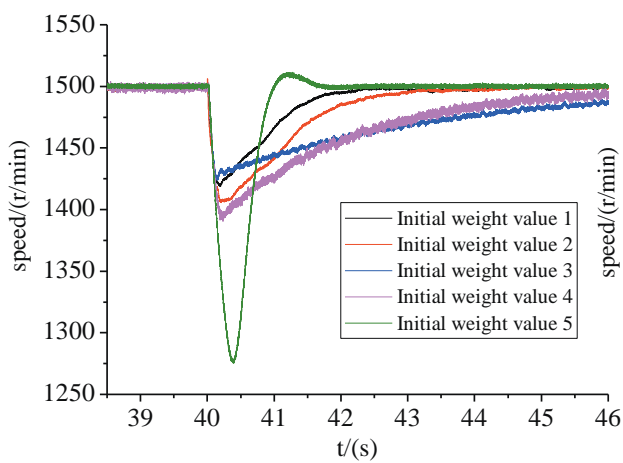

(a)

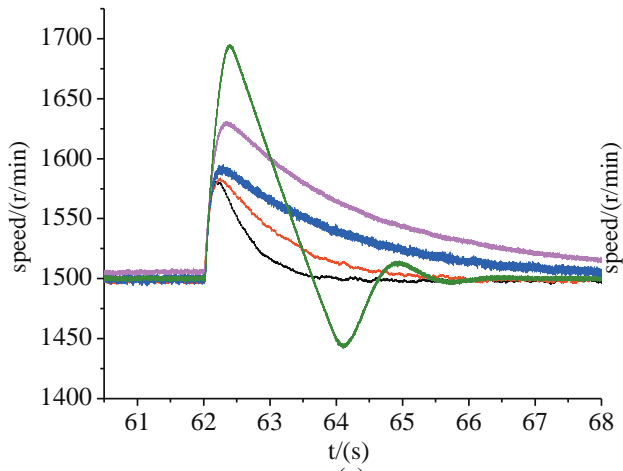

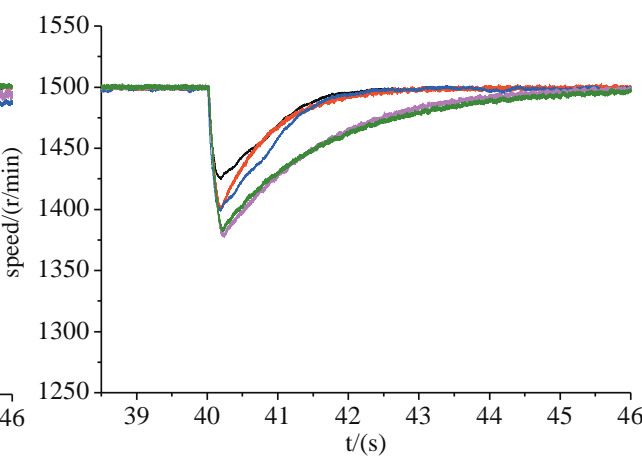

(b)

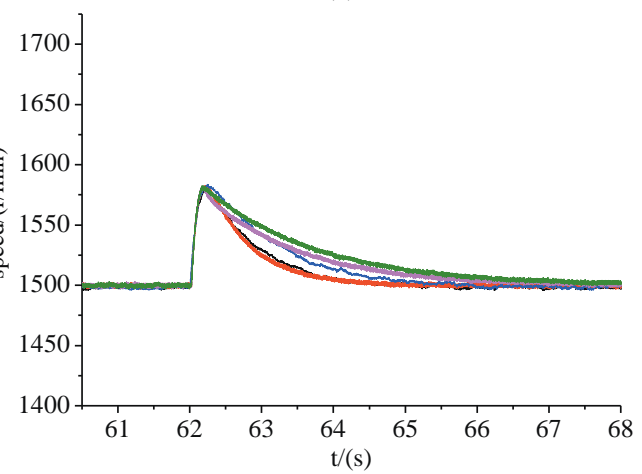

(d)

Figure 8 The comparison of the experimental in speed regulation performance of two intelligent algorithm

\subsection{ADRC controller}

\subsubsection{Offline simulation analysis}

In order to clearly show the control effect of ADRC algorithm, an improved PID controller is designed. The differential signal of the diesel engine speed is obtained by using the tracking differentiator (TD) in ADRC controller to obtain the transition of the speed signal extracted by the controller. The linear combination of the original PID is changed into a nonlinear combination by using the function of $\operatorname{fal}(\varepsilon, \alpha, \delta)$.

The control effect of Traditional-PID, Improved-PID and ADRC controller is analyzed by starting, accelerating, suddenly add $100 \%$ load and load decrease. The simulation process is that after the diesel engine is started, the idle speed (700/min) will be stable for a period of time, and then it will accelerate to the rated speed 
(1500r/min), suddenly add $100 \%$ load at 40 s and then decrease all load at 60 s.

Figure9 shows the simulation result of engine speed variation of three different algorithms, which the case (b) (c) (d) is the enlarged partial detail of Figure (a). The control effect of Improved-PID that using differential tracker (TD) is slightly better than the traditional PID, indicated that the function of differential parameter in PID can be improved by using the TD and the control function can be adjusted by non-linear combination established by function $\operatorname{fal}(\varepsilon, \alpha, \delta)$ according to the speed error, which the speed control performance is improved effectively. However the ADRC controller is established by ESO, TD and NLSEF, which the engine speed and rack displacement as input parameters for ESO to get engine running state and at the same time the differential signal of target speed is extract by TD, and then the NLSEF is used to calculate the control parameter based on the above signal.
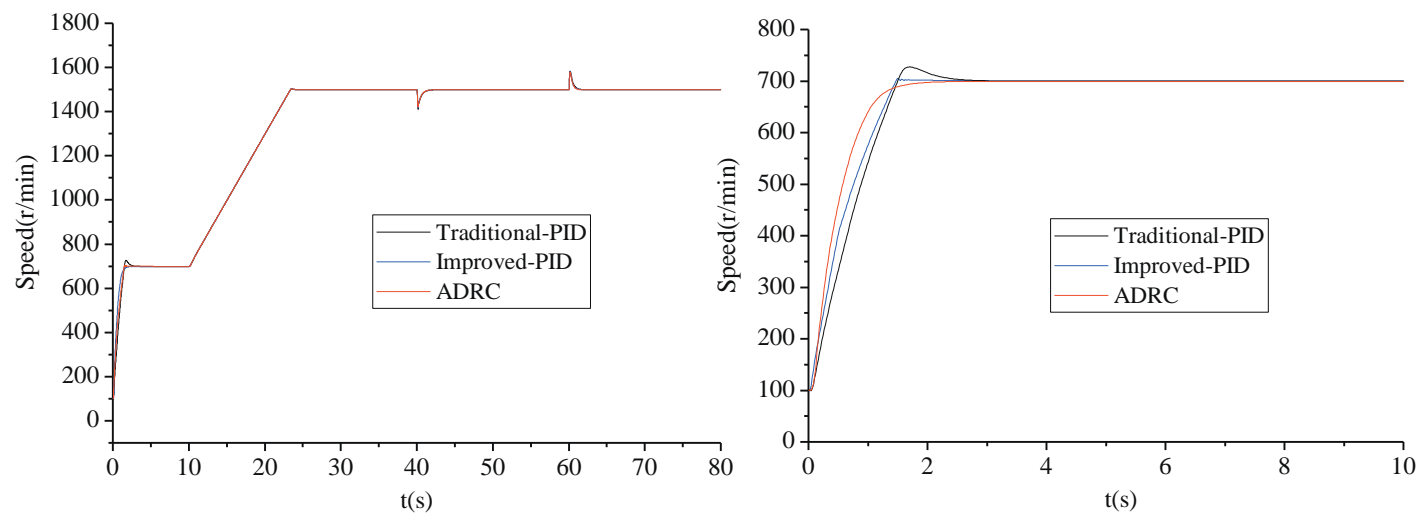

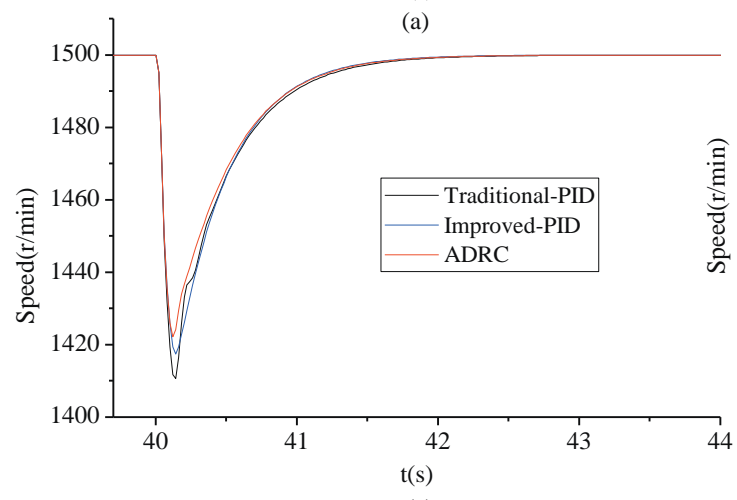

(c)

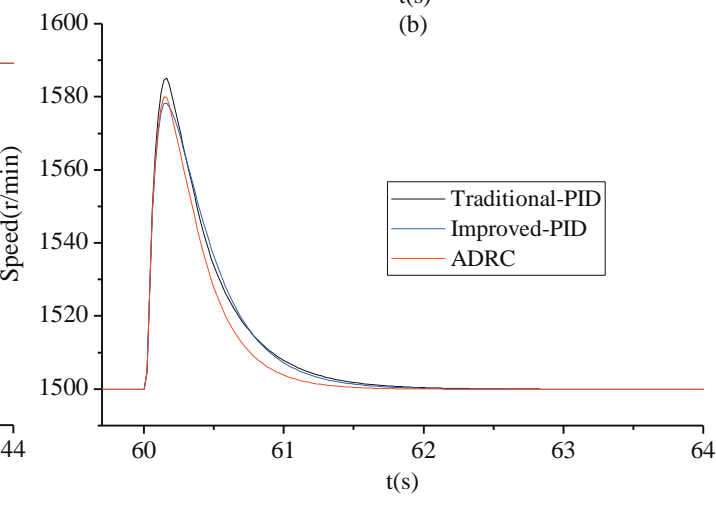

(d)

Figure 9 The comparison of the engine speed of three algorithms in simulation mode

The advantages of ADRC controller cannot be fully highlighted because in simulation condition the engine simulation model has good linearity and small external disturbance. In the next section the control effect of ADRC and Improved-PID is compared by applying an external disturbance to the system on a semi-physical platform.

\subsubsection{Engine matching test verification}

In the machine experiment, the open loop control is adopted in the start-up stage, and the intelligent control algorithm is effective when the idle speed is reached. DS1103 is used as the main controller, and the algorithm is downloaded, and the traditional PID, improved PID and ADRC control algorithms are verified respectively. As shown in Figure11, the speed curve of $100 \%$ of the load mutation in the three control algorithms is shown. 


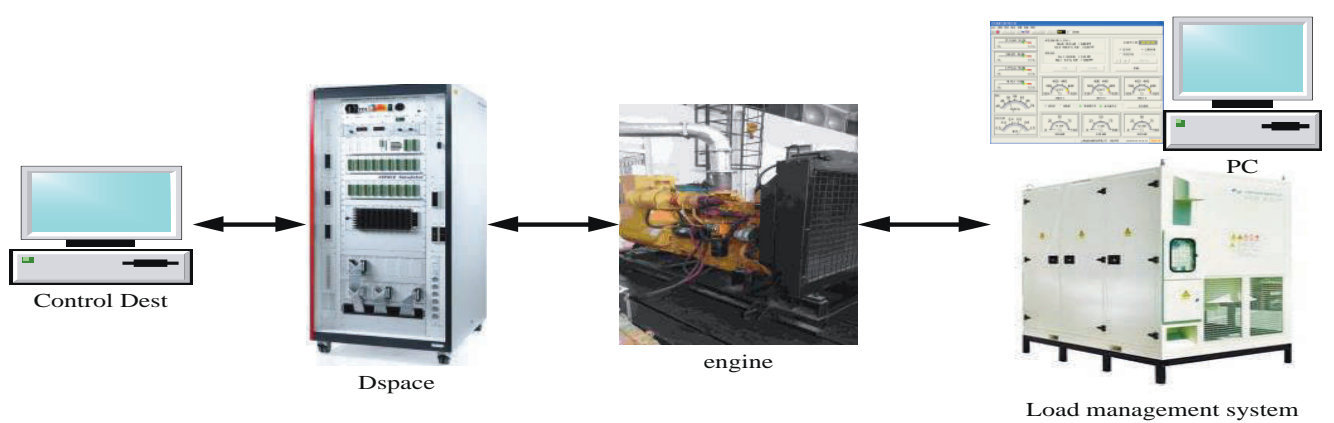

Figure 10 Bench test system

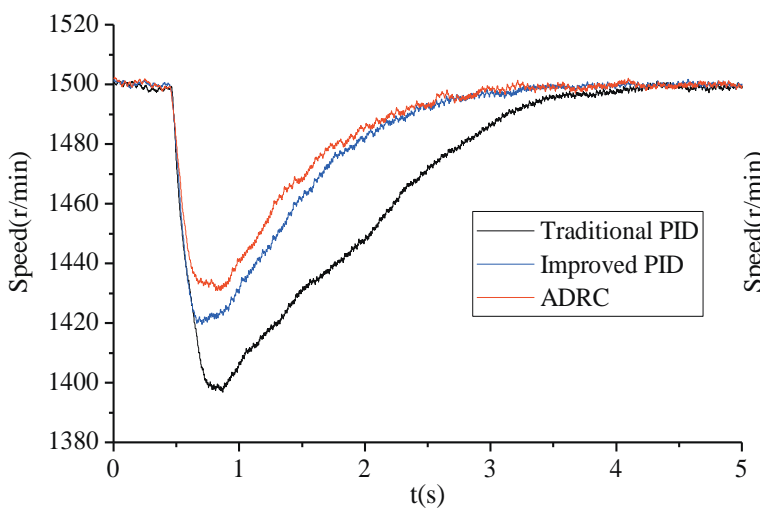

(a)

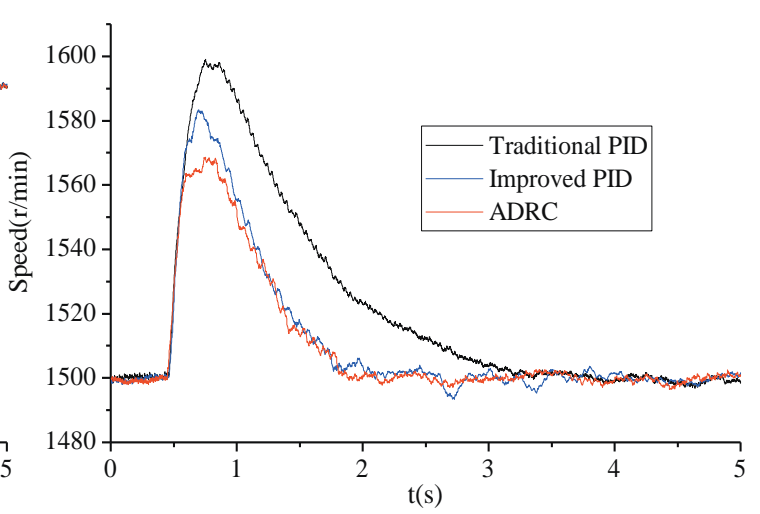

(b)

Figure 11 The comparison of the engine speed of three algorithms in the condition of Sudden $100 \%$ load and reduction, case (a) (b) is the experimental result.

Table 3 Compare for the Experimental Results of Speed Governing Performance

\begin{tabular}{ccccccc}
\hline & \multicolumn{2}{c}{ Traditional PID } & \multicolumn{2}{c}{ Improved-PID } & \multicolumn{2}{c}{ ADRC } \\
\cline { 2 - 6 } & Sudden add & 100\% load & Sudden add & 100\% load & Sudden add & 100\% load \\
& 100\% load & reduction & 100\% load & reduction & 100\% load & reduction \\
\hline Peak speed(r/min) & 1399 & 1597 & 1422 & 1582 & 1431 & 1568 \\
Transient rate (\%) & 6.7 & 6.5 & 5.2 & 5.4 & 4.6 & 4.5 \\
Stable time(s) & 3.02 & 3.11 & 2.04 & 1.68 & 1.98 & 1.45 \\
\hline
\end{tabular}

Compared with semi-physics, the diesel engine has more nonlinearity and delay property is obvious that the same PID parameter can hardly meet the demand of steady and transient state. The increase of perturbation makes it difficult for traditional PID to obtain useful differential signals for differential extraction.

The output of the controller depends on the speed deviation, which is automatically adjusted by function $f a l(\varepsilon, \alpha, \delta)$, and it is effective to distinguish the usefulness of the differential signal extracted by ESO in the steady and transient state. This helps to reduce the interference. The ESO signal $\mathrm{Z}_{3}$ is used as a correction to observe the change of the diesel engine state according to the input signal and make it close to the load change. This is effective for improving the control effect of loading and unloading process. The control effect is shown in Figure11.

\section{Conclusions}

A RBF-PID control algorithm for diesel engine speed regulation is presented in this paper. The controller can adjust the parameters in real time according to the change of speed. Compared with BP neural network 
algorithm, it does not depend on the initial weight, and has better robustness. The experimental results in Table2 show that compared with traditional PID, the transient rate is decreased about $1.3 \%$ and the stable time is shortened about 1 second.

This paper also designs an ADRC controller, and an improved PID controller is developed based on the principle of ADRC and TD module. The effectiveness of traditional PID, improved PID and ADRC control algorithm is verified by off-line simulation, hardware in the loop simulation and experimental test. The results show that in the Improved PID control algorithm, the TD module has a significant improvement on the steady-state and transient performance of the speed regulation. Compared with Traditional PID from Table 3 we can see that the transient rate decreased by $1.5 \%$ under the load adding process, and during load reduction the transient rate decreased by $1.1 \%$; in addition, the stable time also shortened. In the ADRC controller, the ESO module can observe the running state of the engine and provide correction to the controller.

In Table3, compared with the improved PID controller, the transient rate in the loading state is reduced by $0.6 \%$, the load reduction state decreased by $0.9 \%$, and the stability time slightly decreased but not significant.

\section{Further work}

In order to improve the efficiency and cost savings of the ship, the advanced control algorithm can be optimized and transplanted to the next stage of the actual controller. First, the algorithm is optimized to meet the requirements of real time. Secondly, in the conversion process of Simulink program to C code, the code amount should be reduced as much as possible in order to reduce the burden of compilation of single chip microcomputer and finally carry out further experiments. The optimization of engine comprehensive performance index will also be considered in the further works.

\section{References}

Mcgowan D. J., Morrow, D. J., \& Mcardle, M. (2003).: “A digital PID speed controller for a diesel generating set” Power Engineering Society General Meeting (Vol.3, pp.1477 Vol. 3) IEEE.

P. Matić, N. Račić, \& D. Kezić (2009).: "Pidnn for marine diesel main engine speed control”, Naše More Znanstveni Časopis Za More I Pomorstvo, 56(5-6), 193-201.

Roy, S, O. P. Malik, \& G. S. Hope.: "An adaptive control scheme for speed control of diesel driven power-plants." Energy Conversion IEEE Transactions on 6.4(1991):605-611.

Roy, S., O. P. Malik, \& G. S. Hope.: "Real-time test results with adaptive peed controllers for a diesel prime-mover." IEEE Transactions on Energy Conversion 8.3(1993):499-505.

Karray, F., \& E. Conrad.: "Design of intelligent controllers for electronic speed regulation of a diesel engine" First International Conference on Knowledge-Based Intelligent Electronic Systems, 1997. Kes'97. Proceedings IEEE, 1997:607-616 vol.2.

Ha, J. S., \& S. J. Oh.: "Design of an Intelligent Speed Control System for Marine Diesel Engines" Transactions of the Institute of Electrical Engineers of Japan C 21.4(1997):37-43.

Song, H. K., Lee, S. H., \& Goetinck, P. F. (2006). :"Application of CMAC Neural Network \& PID Control on the Speed Control System of Diesel Engine" Intelligent Control and Automation, 2006. WCICA 2006. The Sixth World Congress on(Vol.1, pp.2840-2844). IEEE.

Lei, H. M., \& Hong,W. C. (2007). :'Research on double-pulse h-infinity speed governor for diesel engine of ship power station" Control Theory \& Applications, 24(2), 283-288.

Zhang, G. C., \& G. Ren.: "Research on ship speed regulator using on-line adaptive error model." Chinese Internal Combustion Engine Engineering 30.1(2009):88-92.

Zhang, G. C., \& G. Ren.: "Research on ship diesel engine speed regulator using on-line learning and self-tuning 
error model." Transactions of Csice 27.3(2009):259-264.

Zhang, J., Lan, H. H., \& Sun, Y. (2011). :'Study on the speed control of diesel engine based on neural sliding mode control” Advanced Materials Research, 299-300, 1190-1193.

Mcgowan, D. J., Morrow, D. J., \& Fox, B. (2006). : "Integrated governor control for a diesel-generating set" IEEE Transactions on Energy Conversion, 21(2), 476-483.

Mohammed, N. F., Ma, X., \& Song, E.(2013). : "Tuning of PID controller for diesel engines using genetic algorithm"

Mcgowan, D. J., Morrow, D. J., \& Fox, B. (2008). : "Multiple input governor control for a diesel generating set" IEEE Transactions on Energy Conversion, 23(3), 851-859.

Zhang, Y., Li, S., Lu, G., \& Zhou, Y. (2012). : “A fuzzy self-tuning PID control system of adjustable speed diesel generator" International Conference on Systems and Informatics (pp.619-622). IEEE.

Chen, A. (2010).: "Speed Regulator of Diesel-Generator Based on Model Free Adaptive Control” Intelligent Systems (Vol.3, pp.193-196). IEEE.

Shi, Y., Zhang, L. Y., Sun, J., \& Zhang, H. G.(2013). : "Research on the speed of diesel engine based on improved bp neural network controller" Applied Mechanics \& Materials, 281(1), 105-111.

Shi, Y., Qi, Z. D., Sun, J., Zhang, H. G., \& Shen, Y. L. (2013).: "Research on the control of rack position actuator and marine diesel engine speed based on bp neural network" Chinese Internal Combustion Engine Engineering, 34(4), 42-47.

Sun JB,\& Guo C.: "Simulation of large low speed two-stroke diesel engine propulsion system and design of self-adaptive governor". System Simulation and Scientific Computing, Vols1 and 2, 1160-1164

Mesbahi, E. (2003). Neuro-governor.: “a neural adaptive controller for diesel engines" Control \& Intelligent Systems, 31(3).

Girosi, F., and T. Poggio: "Networks and the best approximation property." Biological Cybernetics 63.3(1990):169-176.

Zadeh, A. G., Fahim, A., \& El-Gindy, M. (1997).: "Neural network and fuzzy logic applications to vehicle systems: literature survey" International Journal of Vehicle Design, 18(2), 132-193.

Pan, Weigang, and F. Chen.: "Design of Ship Main Engine Speed Controller Based on Fuzzy Adaptive Active Disturbance Rejection Technique." International Conference on Intelligent System Design and Engineering Application IEEE Computer Society, 2010:586-589.

Xiao, Hairong, W. Pan, and Y. Han.: "Design of Ship Course Controller Based on Genetic Algorithm Active Disturbance Rejection Technique" Advances in Computer Science, Environment, Ecoinformatics, and Education. Springer Berlin Heidelberg, 2011:232-236.

Pan, Weigang, H. Xiao, and C. Wang.: "Design of Ship Course Controller Based on Optimal Active Disturbance Rejection Technique" IEEE International Conference on Automation and Logistics IEEE, 2010:476-481.

Zhou, Yingbing, W. Pan, and H. Xiao. "Design of ship course controller based on fuzzy adaptive active disturbance rejection technique." 214(2010):232-236.

Pan, Weigang and H. Xiao.: "Design of Diesel Engine Adaptive Active Disturbance Rejection Speed Controller" Open Automation \& Control Systems Journal 7.1(2015):1429-1433.

Pan, Weigang, et al.: "Design of Ship Main Engine Speed Controller Based on Expert Active Disturbance Rejection Technique" Communications in Computer \& Information Science 214(2010):528 - 532.

Pan, W. G., and Y. B. Li.: "Application and simulation research of optimal active disturbance rejection controller in marine main engine" Chinese Internal Combustion Engine Engineering 33.5(2012):74-78.

Kang, E., S. Hong, and M. Sunwoo.: "Idle speed controller based on active disturbance rejection control in diesel engines" International Journal of Automotive Technology 17.6(2016):937-945. 